\title{
Establish Hospice \& Palliative Care Program and Management System with Trans- Disciplinary Team: a Program Manager Perspective with Chinese National Pilot Studies
}

Zhang Ze-Tao ${ }^{1,2}$, Zhu Yi², Wang Na-Ning'2, He Zhao-Ping ${ }^{3}$, He Yu-Ling ${ }^{4}$, Zhao Jian-Ping ${ }^{2}$

${ }^{1}$ Beijing Haidian Hospital, Hospice Palliative Care Unit, Beijing, China

${ }^{2}$ Hospice Palliative Care Alliance of China Foundation, MDPana Research Institute, Eagleville, PA, United States

Presenting \& Corresponding Author: Zetao Zhang Email: ztzhang@msn.com

${ }^{3}$ Ovation Health International, MDPana Research Institute, Wilmington, DE, United States

${ }^{4}$ Ovation Health International, MDPana Research Institute, Copenhagen, Denmark

\begin{tabular}{|c|c|}
\hline & $\begin{array}{l}\text { - Network Development } \\
\text { - Training } \\
\text { - Care Management }\end{array}$ \\
\hline Goal & $\begin{array}{l}\text { - Leverage HPCI to connect patients in need } \\
\text { - Build skills in primary palliative care services } \\
\text { - Support care management by means of standard protocols } \\
\text { and training }\end{array}$ \\
\hline oproach & $\begin{array}{l}\text { - Partner with other players } \\
\text { - Train Care Teams in various settings } \\
\text { - Develop and implement standard protocols }\end{array}$ \\
\hline
\end{tabular}

China is facing a rapidly aging population, and the number of elderly suffering from terminal illnesses has also escalated correspondingly. From the perspective of the national pilot programs and studies the authors participated in, it is both imperative and urgent to accelerate the development of hospice palliative care and service system. In view of the growing demand for end-of-life care services in the hospital, hospice and community. The author established a national project to initiate the "Hospice Palliative Care Initiative"(HPCI). Launched in 2016, the project aims at improve the quality of end-of-life care for terminally ill patients and their family members, enhance the capacity of service providers in the delivery of end-of-life care, as well as raise public awareness. The HPCI is a multi-disciplinary, multi-institutional and cross-sectoral collaboration to help enhance end-of-life care in China with special emphasis on the interface between social and medical systems. Service models are being developed and shaped to provide holistic support to terminally-ill elders in the hospital, hospice, community and elderly homes. The goal is to enable the Chinese older people to have informed choices of care and have an improved quality of life.

Background: In 2016, a hospice palliative care unit was initiated in Beijing Haidian Hospital (aka Haidian Section of Peking University Third Hospital), which was the first pilot study program in Beijing, also first Hospice Palliative Care Haidian Initiative(HPCHI) program of HPCI in China. Challenges of implementing the program and the process to facilitate change in healthcare and society culture in China.

Methods: This Hospice Palliative Care Haidian Initiative(HPCHI) program has been partnered with global organizations Ovation Health International (OHI) and Hospice Palliative Care Alliance of China Foundation (HPCAF) at beginning of the program development in order to streamline the program design and establish SOPs(standard operating procedures), the STOF business model with sustainability, social responsibility \& ethics was taken. Some strategies were adopted to improve the construction of subject, it included the status quo of subject development analyzed with SWOT theory, development objectives formulated according to SMART rules, PDCA rules employed to promote quality improvement of service system, KPI evaluation system established to improve work efficiency. The program was operated by a TransDisciplinary Team(TDT) led by a Program Manager which is the first of these kinds of team structure in China, including the education, research and translational groups, MDT physicians, nurses, pharmacists, technicians, social workers, psychologic counsellors, chaplains and volunteers.

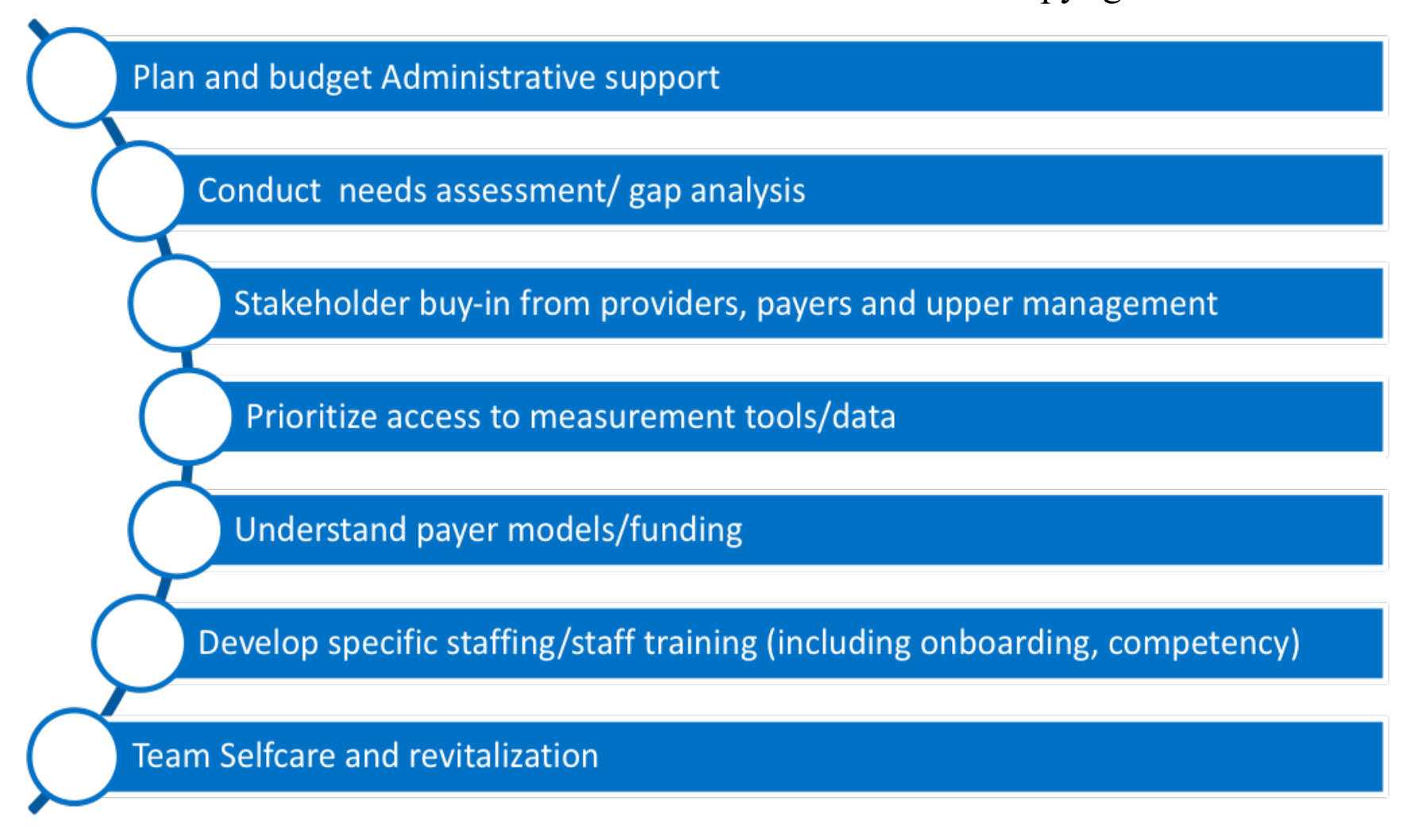

Results: The implementation of the program has been full of obstacles, encompassing the lack of trained professionals, limited financial resources, higher staff turnover, and professional misunderstanding and public rejection. Now HPCHI upgraded to a National Pilot model of China, sponsored by Chinese National Health Commission and Ministry of Civil Affairs, the trial program team led by the program manager, has overcome core barriers/challenges in team building, team enablement, resource supporting, public education, and society awareness to keep the program growth.

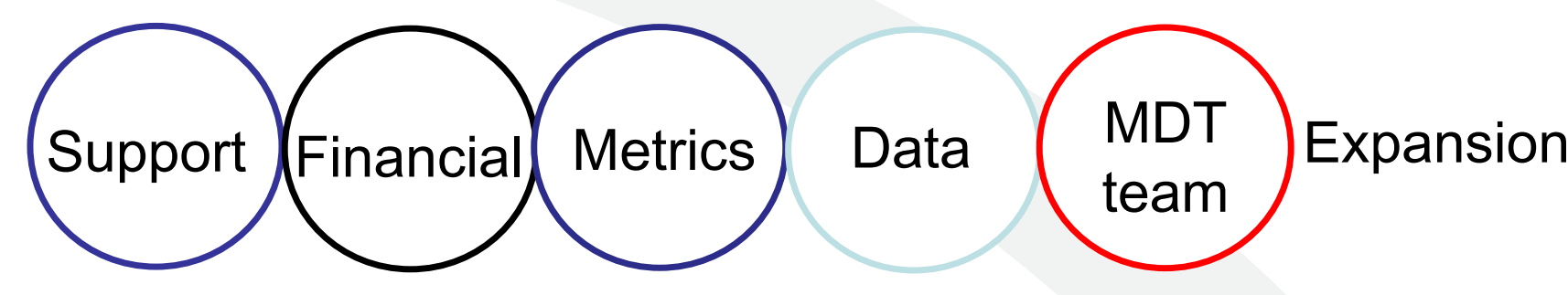

Clarify Program Manager's Value Propositions:

1. Support from leadership is key to sustainability

2. Financial viability must be addressed with shareholders

3. Define measurable Metrics appropriate for the care setting and aligned with the organization's strategy

4. Refer to Metrics to collect data, use data to prove value and cost saving

5. Palliative care requires an interdisciplinary team (regardless of ability to generate revenue)

6. Plan expansion to community settings with collaboration and careful resource planning

Key role: collaborated with Partners (OHI, HPCAF) to Streamline the program design and establish SOPs :

1. Patient Admission Criteria in Admission to inpatient hospice ward

2. Procedure of care transition between hospice ward and other care settings

3. Hospice Patient Care Management Workflow

4. Patient Assessment and Care Plan Evaluation

5. Family Meeting Setup and Meeting Minutes

6. Process to Start Conversation of DNR and living Will

7. Review of Clinical Pathway for palliative care patients

8. Grief counseling service to family members of deceased patients

Conclusions: The lessons learned from the experience of developing the first hospice palliative care program led by the Program Manager in China will be presented. Particularly, barriers and challenges, both modifiable and non-modifiable, will be shared as well as facilitating factors. The authors will share the pathway for program manager of pilot studies to partner across disciplines, with policy makers, and in research, education and practice. This helps the team in the creation of new knowledge and in continuing to establish the evidence-based value of hospice palliative care combined social and medical systems in China. 